\title{
Especie nueva de hidracárido del género Piona (Acari: Hydrachnidia: Pionidae) de los canales de Xochimilco, México
}

\author{
New species of water mites of the genus Piona (Acari: Hydrachnidia: Pionidae), from Xochimilco's \\ water channel, Mexico
}

\author{
Carlos Humberto Marín-Hernández y Cristina Cramer-Hemkes* \\ Laboratorio de Acarología “Anita Hoffmann”, Departamento de Biología Comparada, Facultad de Ciencias, Universidad Nacional Autónoma de \\ México, 04510 México, D.F. México. \\ *Correspondencia: cch@fciencias.unam.mx
}

\begin{abstract}
Resumen. Se describe una especie nueva de hidracárido (Hydrachnidia: Pionidae) perteneciente al género Piona, recolectada en canales de la zona lacustre de Xochimilco, México, D. F. Éste es el primer registro de la familia y del género para la ciudad de México.
\end{abstract}

Palabras clave: ácaros acuáticos, ácaros dulceacuícolas, Hydracarina, Hydrachnellae.

\begin{abstract}
A new water mite species is described, belonging to the genus Piona (Hydrachnidia: Pionidae), collected from a canal at Xochimilco, México City, Mexico.This represents the first family and genus record for México City.
\end{abstract}

Key words: Hydracarids, Hydracarina, Hydrachnellae.

\section{Introducción}

La familia Pionidae agrupa ácaros acuáticos que se distribuyen principalmente en la región holártica. Se localizan en ambientes lénticos y lóticos, con excepción de las aguas termales. Originalmente fue descrita por Thor (1900) y a la fecha posee 5 sinónimos: Curvipedidae Thor, 1900; Acercidae Thor, 1909; Foreliidae Thor, 1923; Nautarachnidae Walter, 1925 e Hydrochoreutidae Viets, 1942.

Dentro de esta familia, se han incluido 6 subfamilias: Huitfeldtiinae, Pioninae, Nautarachninae, Tiphyinae, Foreliinae y Astacocrotoninae. El género típico Piona agrupa la gran mayoría de las especies. La distinción de subgéneros de Besseling (1959) se basa en la presencia o ausencia de la concavidad en la placa genital de los machos y en las modificaciones ventrales y tamaño de las uñas del tarso III que éstos presentan. La propuesta por Cook (1960), que se basa en la presencia, número y posición de las placas acetabulares de las hembras, establece un sistema confuso, cuya aplicación asigna diferentes subgéneros a individuos hembra y macho de una especie y/o población. Para subsanar esta problemática, Cook (1974) replantea este ordenamiento, proponiendo 3 subgéneros diferentes con base en los caracteres de los machos:

Recibido: 23 noviembre 2007; aceptado: 06 julio 2008
Piona, sin concavidad genital, y tarso de la pata III con una uña larga y una corta; Conglopiona. sin concavidad genital y 2 uñas cortas en el tarso de la pata III; Dispersipiona, con una concavidad genital profunda y 2 uñas cortas en el tarso de la pata III.

En México, el género Piona se ha citado de los estados de Campeche, México, Nuevo León, Oaxaca, Tabasco, Tamaulipas y Veracruz (Cook, 1980, Cramer, 1980, Otero, 1986, Ramírez, 1994), con 5 especies descritas hasta el momento: Piona (Piona) triangularis Wolcott, 1902; P. (Tetrapiona) sudamericana rotunda Viets, 1910; P. bromada Cook, 1980; P. neoacutidens Cook, 1980; P. mexicana Cook, 1980.

\section{Materiales y métodos}

Los ejemplares de la especie que se describe en este trabajo fueron recolectados en los canales de la zona lacustre de Xochimilco, en el embarcadero Belem, delegación Xochimilco, ciudad de México, (Cda. de Violeta s/n, Barrio Belem) a una altitud de $2439 \mathrm{~m} \mathrm{snm}$, temperatura de 15 a $20{ }^{\circ} \mathrm{C}$ y profundidad promedio de $140 \mathrm{~cm}$; generalmente asociados con fondo arenoso y en ocasiones con plantas de Ceratophylum demersum, Egeria densa y Eichhornia crassipes. Para recolectar, separar, fijar y montar los organismos se usaron las técnicas y 
metodología descritas por Cramer (1988). Se utilizaron redes de malla fina, cubetas de 12 y 20 litros, charolas de plástico de fondo blanco y bolsas de plástico. El sustrato arenoso se obtuvo con ayuda de una cubeta, revisando directamente en las charolas y sacando los ácaros con auxilio de un gotero. Asimismo, se recorrió la red a lo largo de las orillas para obtener la vegetación asociada, la cual se lavó y filtró. Los hidracáridos se fijaron y conservaron con líquido de Koenike y se montaron en gelatina glicerinada para realizar el estudio taxonómico correspondiente. Las medidas de los ejemplares tipo se obtuvieron con ayuda de un ocular micrométrico y reglilla y están dadas en micrones ( $\mu$ ). Las abreviaturas son las de Cramer (1988).

El holotipo macho, recolectado en el embarcadero Belem, Xochimilco, México, D. F. el 04.X.2001, 3 paratipos, $\lesssim \widehat{\partial} \mathrm{y} q$ del mismo sitio y fecha de colecta que el holotipo, 1 ㅇ del 21.III.2002, 1 đ del 02.IV.2002 y 2 đo del 09.IV.2002. Marín-Hernández C. Col. El holotipo

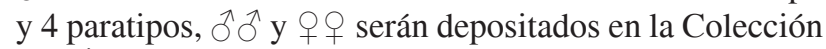
de Ácaros Acuáticos del Laboratorio de Acarología de la Facultad de Ciencias de la UNAM y 1 paratipo $\widehat{\sigma}$ será depositado en la Colección Nacional de Ácaros y Arácnidos del Instituto de Biología de la UNAM.

\section{Descripción}

\section{Piona Koch, 1842}

Nesaea Koch, 1836. Deutschlands Crust., Myr. und Arach. 5: 21 .

Piona Koch, 1842. Übersicht des Arachnidensystems, Nürnberg 3: 13.

Curvipes Koenike, 1891. Zool. Anz. 14: 20.

Especie tipo: Piona ovata Koch, 1842

Diagnosis: véase Cook (1974) y Smith (1976)

Hábitat: ambientes lóticos y lénticos: arroyos, lagos, lagunas y charcas temporales.

Distribución: principalmente en la región holártica (Cook, 1976), aunque se conocen algunas especies de América Central y del Sur, África, Australia y Nueva Zelanda (Smith y Cook, 1999).

\section{Piona amimitli n. sp. (Figs. 1-6)}

Macho. Largo del idiosoma 969-1223; largo del margen anterior de la coxa I a la porción terminal de la placa genital 687-772, ancho 706-856; CI con los bordes internos relativamente largos los cuales se tocan en la parte media y se separan hacia la parte posterior; epimeroglandularia I ligeramente fusionada; extremos anteriores de las CI sobresalen de la parte anterior del gnatosoma; apodemas posteriores de las CI y CII poco desarrollados; CIII y CIV claramente fusionadas entre sí y con la placa acetabular; líneas de sutura entre la tercera y cuarta coxa incompletas; borde externo asociado a la inserción de la pata IV bien desarrollado; placa genital extendida lateralmente alcanzando la proyección posterior de las coxas IV; placa genital 320-386 de ancho; gonoporo amplio con 47-104 de largo, 94-151 de ancho, con una concavidad o depresión profunda en la parte posterior y 18-28 acetábulos por placa; epimeroglandularia 2 libre (Fig. 1); largo de los artejos del pedipalpo: Tr, 50-71; Fe, 179-226; Ge, 88-104; Ti, 226-273; Ta, 104-122; Fe con 1 seda grande en la parte media y 1 en la parte terminal del extremo distal; lado ventral de la tibia con 2 tubérculos bien desarrollados con una seda pequeña cada uno; tibia y tarso ligeramente más angostos que el resto, la figura 6 muestra las proporciones y la quetotaxia del pedipalpo; base del gnatosoma 226 de largo; longitudes dorsales de los artejos de las patas: I-Tr: 66-94; I-Fe: 122-188; I-Fe: 160-198; I-Ge: 263-292; I-Ti: 320-395; I-Ta: 254-292; II-Tr: 75-94; II-Fe: 132-188; IIFe: 169-207; II-Ge: 282-356; II-Ti:320-414; II-Ta: 245311; III-Tr: 75-113; III-Fe: 112-160; III-Fe: 104-141; III-Ge: 216-254; III-Ti: 320-367; III-Ta: 132-160; IV-Tr: 141-169; IV-Fe: 151-170; IV-Fe: 132-151; IV-Ge: 235273; IV-Ti: 338-395; IV-Ta: 263-361; II-Ti con 7-14 sedas natatorias; III-Ti con 10-13 y IV-Ti con 11-14. Pata III con la uña dorsal más cortas y otra en forma de gancho como se ilustra en la figura 4. Pata IV del macho, con las modificaciones propias de la especie, genua con marcada concavidad ventral y sedas engrosadas asociadas a la misma, como en figura 5.

Hembra. Largo del idiosoma 1409-1788; largo del extremo anterior de la CI a la parte posterior de la placa genital 809-969; epimeroglandularia I libre; bordes asociados a la inserción de la cuarta coxa bien desarrollados; largo de la placa acetabular 104-179, ancho 376-447; gonoporo largo 160-339; (Fig.2); 13-42 acetábulos de cada lado; extremo anterior de la CI proyectándose más allá del gnatosoma; apodemas posteriores de las CI y CII relativamente desarrollados; largo de los artejos del pedipalpo: Tr, 5667; Fe, 207-245; Ge, 85-104, Ti, 235-273; Ta, 113-132; Fe igual al macho; Ti con tubérculos bien desarrollados (Fig. 6); largo de la base del gnatosoma 112; longitudes dorsales de los artejos de las patas: I-Tr: 75-94; I-Fe: 141-179; IFe: 169-198; I-Ge: 301-339; I-Ti: 339-376; I-Ta: 254-301 (Fig.3); II-Tr: 94-122; II-Fe: 160-188; II-Fe: 169-235; IIGe: 254-367; II-Ti: 358-414; II-Ta: 282-311; III-Tr: 75122; III-Fe: 151-188; III-Fe: 188-207; III-Ge: 339-376; III-Ti: 376-423; III-Ta: 292-301; IV-Tr: 141-216; IV-Fe: 188-235; IV-Fe: 263-282; IV-Ge: 395-423; IV-Ti: $433-$ 461; IV-Ta: 273-292; II-Ge con 7-8 sedas natatorias, II-Ti con 7-10; III-Ge con 10-13, III-Ti con 8-13; IV Ge con 11-13 y IV-Ti con 8-14. 


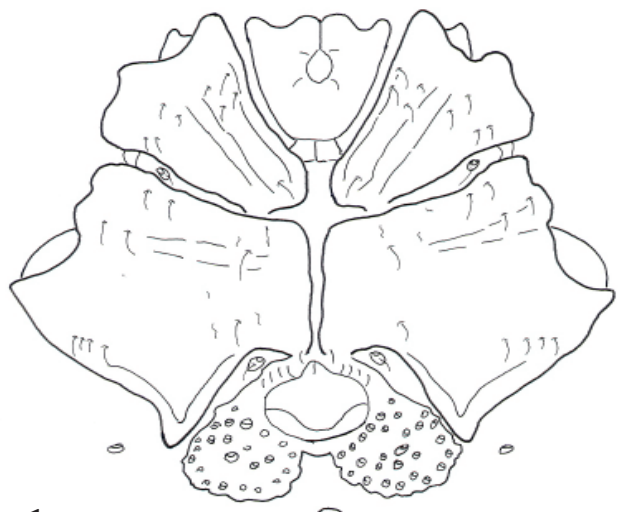

1

(1D)
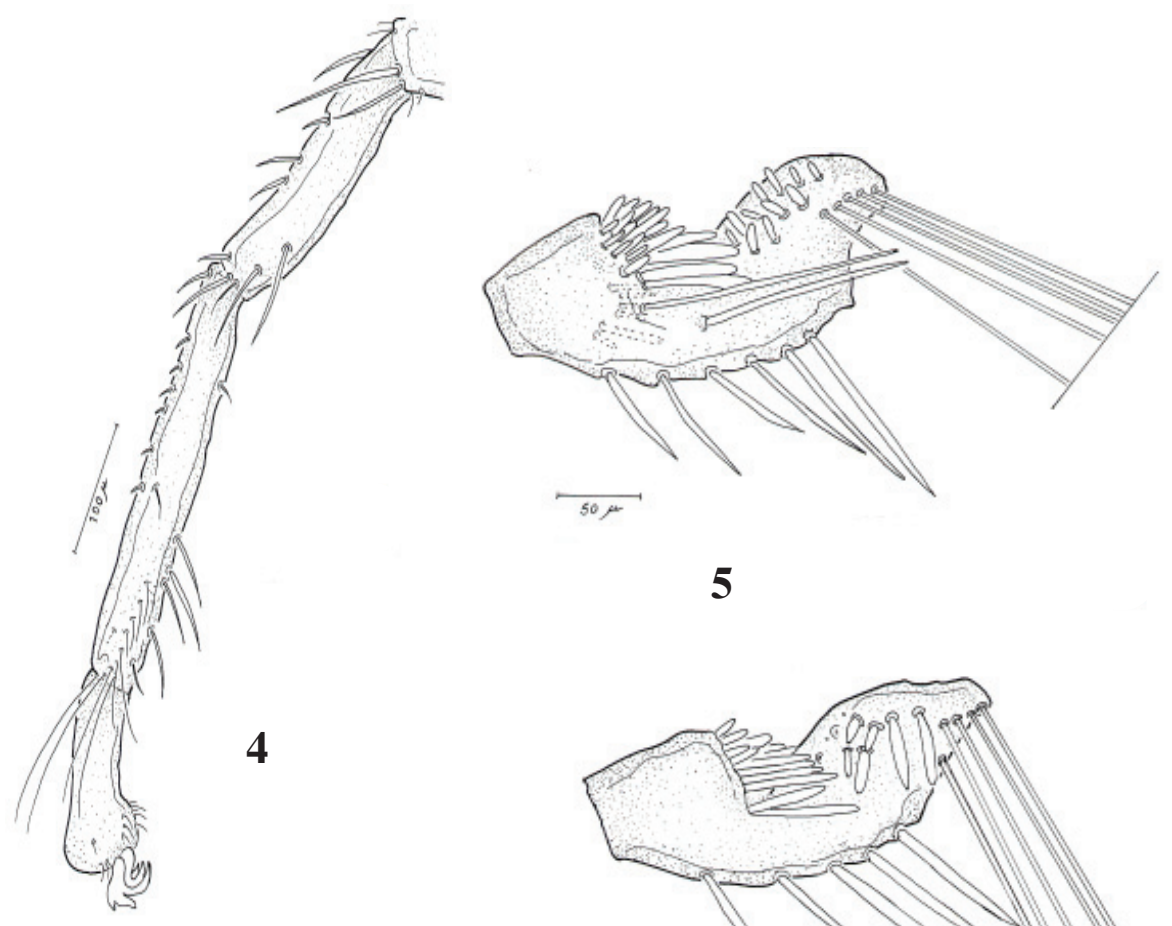

5
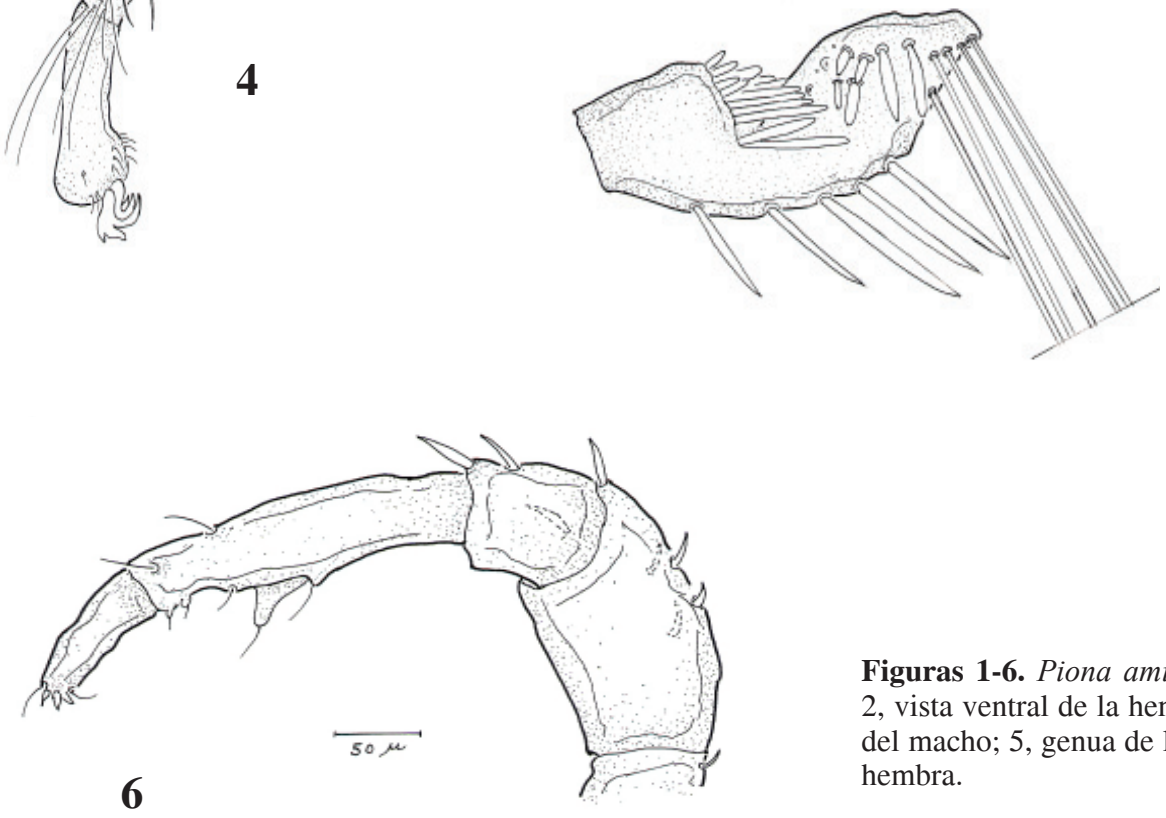
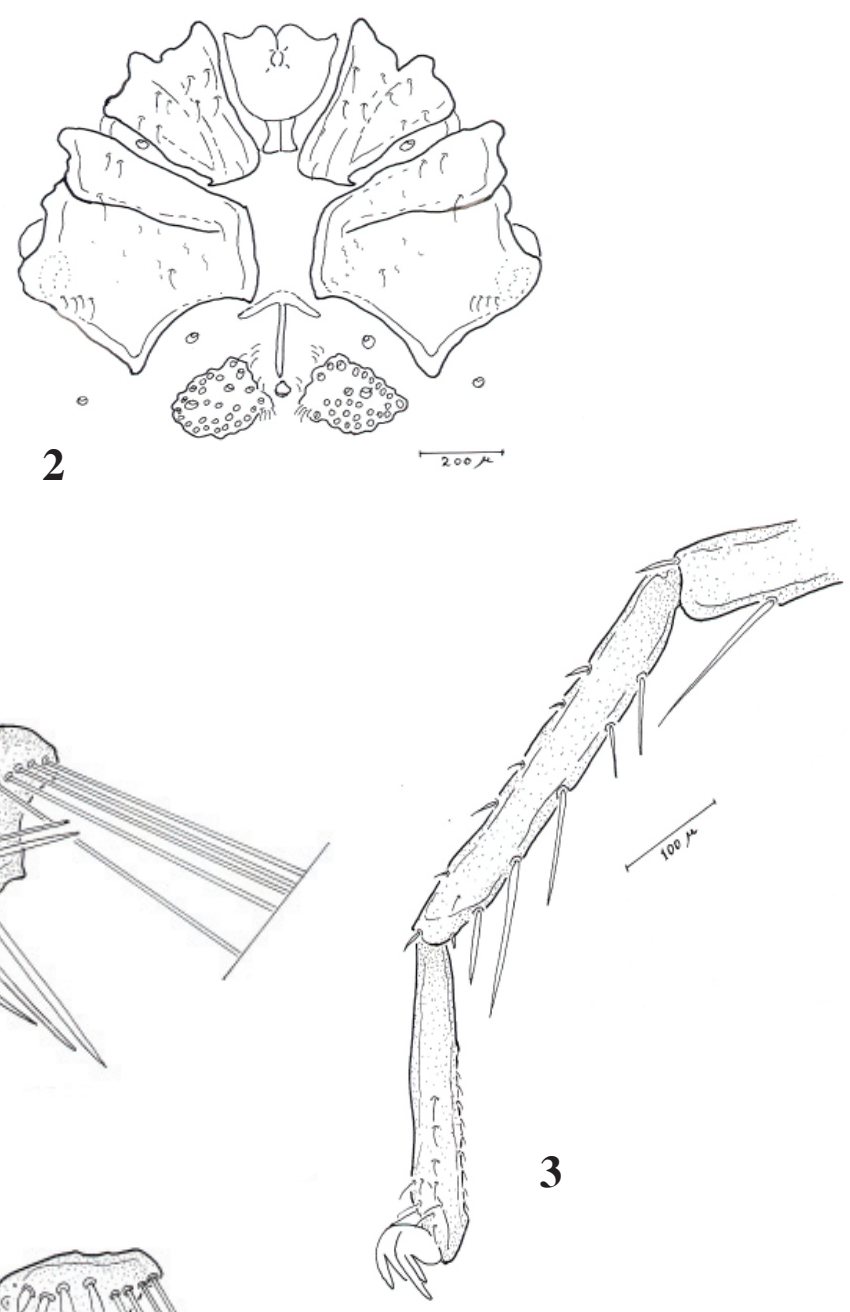

Figuras 1-6. Piona amimitli n. sp. 1, vista ventral del macho; 2 , vista ventral de la hembra; 3 , pata I de la hembra; 4, pata III del macho; 5, genua de la pata IV del macho; 6, pedipalpo de la hembra. 


\section{Resumen taxonómico}

Los ejemplares de la especie descrita son típicos de ambientes lóticos y se encuentran distribuidos en varios canales del lago de Xochimilco.

Etimología. Amimitli, el dios xochimilca protector de los pescadores; del nahuátl mitl " flecha" y atl "agua", "cazador del agua”, relacionado con el hábito depredador de la especie.

\section{Comentarios taxonómicos}

La especie Piona amimitli, constituye el primer registro de la familia Pionidae y del género para la ciudad de México. Al parecer, su distribución está restringida al área de Xochimilco;sin embargo, es indispensable realizar un mayor número de colectas en áreas aledañas para poder determinar si esta especie es endémica de la zona. Tomando como base los caracteres morfológicos de ésta, y en particular las modificaciones de la pata III del macho, se puede señalar que existe una cercanía con grupos de especies neárticas, especialmente del norte de los Estados Unidos de América. La especie más cercana en relación con las ya conocidas es $P$. coccinea americana, descrita por Marshall de Wisconsin, Alaska y Nuevo México. Tanto esta especie norteña como la que se describe aquí tienen la uña dorsal de la pata III del macho en forma de gancho; sin embargo, en $P$. amimitli esta uña es más corta y el gancho es más abierto; se diferencia también en que la tibia y el tarso de la misma pata son más cortos y el número de acetábulos es menor en cada una de las placas del área genital. Se puede señalar también que en el macho de $P$. interrupta una de las uñas de la pata III se encuentra modificada como en la especie mexicana; sin embargo, el número de acetábulos en la especie estadounidense sobrepasa por mucho los de $P$. amimitli y $P$. coccinea americana. Cook (1974) subdivide Piona en 3 subgéneros, correspondiendo la hembra de esta nueva especie al subgénero típico Piona (s.s.) y el macho (con una depresión en el área genital y uñas características de la pata III) a uno muy distinto de los que a la fecha se han descrito. Este análisis permite evaluar conjuntamente con estudios y descripciones que preceden a ésta, que los caracteres morfológicos de los machos pueden considerarse más "estables", y que los "arreglos" taxonómicos propuestos por diversos autores para Piona continúan siendo sumamente artificiales, en especial si se utilizan caracteres de las hembras. Para ello, es indispensable llevar a cabo el estudio de las larvas, de modo que se pueda conjuntar dicha información con los caracteres que han permitido a partir de los machos, el establecimiento de los diferentes subgéneros. Asimismo, será de gran importancia realizar una revisión del género antes de asignar un subgénero a Piona amimitli.

\section{Agradecimientos}

A la Dra. Anita Hoffmann por sus comentarios al manuscrito, al Dr. David R. Cook por la revisión de los ejemplares y a la Biól. Ma. del Carmen Letechipía por su ayuda en la separación y montaje de los ejemplares.

\section{Literatura citada}

Besseling, A. J. 1959. Nederlandse Hydrachnellae XL. Entomologische Berichten 19:20-23.

Cook, D. R. 1960. Water mites of the genus Piona in the United States (Acarina: Pionidae). Annals of the Entomological Society of America 53:35-60.

Cook, D. R. 1974. Water mite genera and subgenera. Memoirs of the American Entomological Institute 21:860 p.

Cramer, H. C. 1980. Ácaros acuáticos de un arroyo de Avándaro, Méx. Folia Entomológica Mexicana 45:72-73.

Cramer, H.C. 1988. Ácaros dulceacuícolas (Acarida: Prostigmata) del arroyo Peña Blanca en San Francisco Oxtotilpan, México. Tesis, Facultad de Ciencias, UNAM, México, D. F. 269 p.

Otero, C. G. 1986. Contribución al conocimiento de los ácaros acuáticos (Prostigmata: Parasitengona) del sureste de México. Tesis, Escuela Nacional de Ciencias Biológicas, Instituto Politécnico Nacional, México, D. F. 369 p.

Ramírez, B. P. 1994. Taxonomía, distribución y datos ecológicos de los ácaros acuáticos (Acari: Prostigmata) de la presa Marte R. Gómez, Tamps. y río Agualeguas, N. L. Tesis, Facultad de Ciencias Biológicas, Universidad Autónoma de Nuevo León, Monterrey. 145 p.

Smith, I. M. 1976. A study of the systematics of the water mite family Pionidae (Prostigmata: Parasitengona). Memoirs of the Entomological Society of Canada 98:249.

Smith, I. M. y D. R. Cook. 1999. An assessment of global distribution patterns in water mites (Acari: Hydrachnida). In Acarology. IX. Symposia, G. R. Needham, R. Mitchell, D. J. Horn y W. C. Welbourn. (eds.). Ohio Biological Survey. Ohio University, Columbus. p. 109-24. 